\title{
Optimization of train plan for urban rail transit in the multi-routing mode
}

\author{
Lianbo DENG ${ }^{1 *}$, Qiang ZENG ${ }^{1}$, Wei GAO ${ }^{1}$, Song $\mathrm{BIN}^{2}$ \\ 1. School of Traffic and Transportation Engineering, Central South University, Changsha 410075, China \\ 2. China Railway Siyuan Survey and Design Group Co. Ltd., Wuhan 430063, China
}

\begin{abstract}
The train plan of urban rail transit under multi-routing mode can be divided into three parts: train formation, train operation periods and corresponding train counts of each routing in each period. Based on the analysis of passenger's general travel expenses and operator's benefits, the constraints and objective functions are defined and the multiobjective optimization model for the train plan of urban rail transit is presented. Factors considered in the multiobjective optimization model include transport capacity, the requirements of traffic organization, corporation benefits, passenger demands, and passenger choice behavior under multi-train-routing mode. According to the characteristics of this model and practical planning experience, a three-phase solution was designed to gradually optimize the train formation, train counts as well as operation periods. The instance of Changsha Metro Line 2 validates the feasibility and efficiency of this approach.
\end{abstract}

Key words: urban rail transit; multi-train-routing; train plan; multi-objective model; three-phase solution method

(C) 2011 JMT. All rights reserved.

\section{Introduction}

$\mathrm{O}$ ver years, the trains on urban railways in China have to stop at every station along a single long route. The daily train plan depends on the cross-section flow in each operation period [1].

In order to provide service efficiently and to decrease cost effectively, many cities in China have to take the approaches such as train formation adjustment [2] and multi-routing operation [3] to make their rail transit networks more competitive. The traditional train plan of urban rail transit has expanded into more advanced one, which consists of train formation, train operation period and train frequency for each routing [4]. Among these components, the most important determinant of transport capacity is train formation. In the multi-trainrouting mode, the train plan needs to consider not only the heterogeneous distribution of passenger volumes in the time-space, but also the passengers' choices [5-6].

Chang et al. [7] presented a multi-objective optimization model to minimize operation cost and total travel time of passengers. Train stops, service frequency and train count were optimized by fuzzy mathematic pro-

Received Aug. 29, 2011; revision accepted Nov. 17, 2011

*Corresponding author. E-mail: lbdeng@csu.edu.cn (L.B. DENG)

(C) 2011 JMT. All rights reserved

doi: 10.3969/j.issn.2095-087X.2011.04.003 gramming. Bussieck et al. [8] treated the train plan as a train schedule (i.e. a line plan), and determined the number of trains connecting two terminal stations of a serving line in a fixed time interval. Claessens et al. [9] built an integer nonlinear model to maximize the number of direct travelers for the Dutch railway system. Sun et al. [10] investigated the multi-train-routings of urban rail, and proposed a two-phase solution for the maximal seat occupancy rate and the minimal count of train operation periods. Deng et al. [4] considered single train routing based on the analysis of passenger's general travel expenses, and established a multi-objective model with respect to transport capacities, transport organizations, economic benefits and traffic demands. Refs. [1112] studied passenger's choice behavior and flow assignment in the urban rail transit network. Sang et al. [11] presented a graph theoretic framework for the passenger assignment problems that simultaneously encompassed the departure time and the route choice. Tian et al. [12] analyzed the equilibrium properties with invehicle crowding effect and schedule delay cost in a many-to-one transit system.

Although the train plans of urban rail transit are essentially network problems. Unlike national railway network, they are independent of each other to some extent. In this paper, the train plan is targeted on one line with the consideration of the passenger choice. For simplification, passengers transfer is ignored. 


\section{Related concepts on passenger train plan}

Train routing set is expressed as $U=\left\{u_{l}=\left(s_{l}, e_{l}\right) \mid l=\right.$ $\left.1, \cdots, H_{u} ; s_{l}, e_{l} \in S\right\}$ where $s_{l}$ and $e_{l}$ are the two terminals of routing $u_{l}$. The train routing modes include: adjacent routing, nested routing, and the mixed mode (Fig. 1). For smooth train operation and passenger boarding, $H_{u}$ can not be excessive. Among all the routings, the ones running in all operation periods are basic routings whereas others running in peak periods are non-basic ones. Adjacent routings are basic. The long routing of the nested routing mode is a basic one while the short is not. The train plan for the adjacent routing mode can be optimized on a single routing basis. Comparatively, the train plan of the nested routing mode should be optimized according to passenger's choice. Further, the train plan of the mixed mode is a combination of both plans over two routing modes. The train plan optimization of the nested routing mode is the focus in this paper.

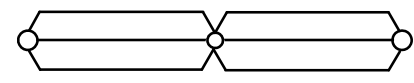

(a) Adjacent routing mode

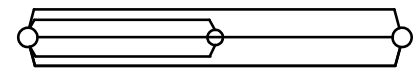

(b) Nested routing mode

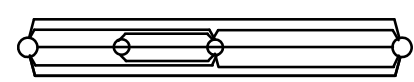

(c) Mixed mode

Fig. 1 Routing modes

Let $\left[T_{s}, T_{e}\right]$ denote the daily operation period of urban rail transit. The train formation is usually unchanged. For the train plan $\Omega, b$ is the train formation length (i.e. number of carriages in a train), and $V$ is the average carrying capacity of carriages. Considering the factors such as station capacity and track length, the feasible formation length is in a range of $[\bar{b}, \overline{\bar{b}}]$, where $\bar{b}$ and $\overline{\bar{b}}$ are the shortest and the longest formation lengths respectively.

According to the fluctuation of passenger flow in one day and the variation of train frequency in $\left[T_{s}, T_{e}\right]$, we can define the train operation period $T_{i}$. Set $T=\left\{T_{i}=\left(t_{i}^{a}, t_{i}^{b}\right) \mid i=1,2, \cdots, H_{t}\right\}$ to be train operation periods, where $t_{i}^{a}$ and $t_{i}^{b}$ denote the start and the end time of $T_{i}$ respectively; $H_{t}$ is the count of train operation periods under the conditions of $t_{1}^{a}=T_{s}$ and
$t_{H_{t}}^{b}=T_{e}$.

The train plan of urban rail transit $\Omega$ determines train formation and the number of trains for each routing and operation period. For simplicity, we only consider the train plan in a sequence of $1,2, \cdots, H_{s}$. The number of trains on routing $u_{l}$ during $T_{k}$ is $d_{k}^{l}$, and train set is $D=\left\{d_{k}^{l} \mid k=1,2, \cdots, H_{t} ; l=1,2, \cdots, H_{u}\right\}$. According to above definitions, $\Omega=\{b, T, D\}$ can denote the train plan of urban rail transit.

\section{Passenger's general travel expenses}

Passenger flow of urban rail transit fluctuates in $\left[T_{s}, T_{e}\right]$. It is relatively steady in certain duration, which is called passenger travel period. $\left[T_{s}, T_{e}\right]$ can be divided into $H_{t}^{0}$ passenger travel periods. The passenger flow from station $i$ to station $j$ in period $T_{k}^{0}$ is $f(i, j, k)$, for $i, j=1,2, \cdots, H_{s}, k=1,2, \cdots, H_{t}^{0}$. The train frequency is unified in one passenger travel period, and one train operation period may include one or more passenger travel periods. If the passenger travel period $T_{k_{1}}^{0}$ is included in train operation period $T_{k_{2}}$, the relationship can be expressed as $T_{k_{1}}^{0} \in T_{k_{2}}$, for $k_{1}=1,2, \cdots, H_{t}^{0}$, and $k_{2}=1,2, \cdots, H_{t}$.

The passenger's general travel expense usually includes ticket fare, travel time and congestion cost.

Ticket fare is a monetary expenditure, which can be expressed as person-kilometers fare rate $p$. Individual ticket fare for passenger flow $f(i, j, k)$ is

$$
P(i, j, k)=p w(i, j)
$$

Travel time includes riding time and waiting time. Riding time is equal to the train running time between the stations, which can be calculated by dividing the distance $w(i, j)$ with the train speed $\gamma$. The riding time for passenger flow $f(i, j, k)$ is

$$
G(i, j, k)=\frac{w(i, j)}{\gamma} .
$$

Waiting time refers to the time period from a passenger's arrival to departure. The waiting time is related to train frequency. When $T_{k_{1}}^{0} \in T_{k_{2}}$, the average waiting time for the flow $f\left(i, j, k_{1}\right)$ is calculated by 


$$
z\left(i, j, k_{1}\right)=\frac{\sigma\left|T_{k_{2}}\right|}{\sum_{u_{l} \in U: u_{l} \subseteq e(i, j)} d_{k_{2}}^{l}},
$$

where $\left|T_{k_{2}}\right|$ is the period length of $T_{k_{2}} ; \sigma$ is a parameter related with the passenger flow distribution, and $\sigma$ is equal to 0.5 for uniform passenger flow.

$f(i, j, k, l)$ is the volume of passengers which select train routing $u_{l}$ in $f(i, j, k)$. Within the passenger travel period $T_{k}^{0}$, the passenger volume on routing $u_{l}$ in the section $(i, i+1) \in E$ is

$$
g(i, k, l)=\sum_{j=i+1}^{e_{l}} \sum_{i=s_{l}}^{i} f\left(i^{\prime}, j^{\prime}, k, l\right) .
$$

We introduce the congestion cost function $y(g(i, k, l))$ to describe the passenger discomfort for routing $u_{l}$ and passenger flow period $T_{k}$.

The time and congestion cost of one passenger should be converted into monetary expenditure. From station $i$ to station $j$ in $T_{k}^{0}$, the individual travel expense is

$$
\begin{aligned}
& C(i, j, k, l)=P(i, j, k)+ \\
& \quad \beta[G(i, j, k)+Z(i, j, k)]+\sum_{i^{\prime}=i}^{j-1} y\left(g\left(i^{\prime}, k, l\right)\right),
\end{aligned}
$$

where $\beta$ refers to the average time value of passengers.

\section{Multi-objective optimization model}

Within the passenger travel period $T_{k_{1}}^{0}$, the train load factor varies at different sections of train routings. From the perspective of transport capacity and operation cost, the load factor is controlled in the sections of passenger flow peak to ensure reasonable overall load factor. The lower limit of the train load factor is denoted by $\varphi_{0}$ ranging from 0.7 to 0.9 , and upper limit is denoted by $\varphi_{1}$ ranging from 1.2 to 1.5 . The minimal and maximal passengers volume of the section are respectively $\varphi_{0} V b$ and $\varphi_{1} V b$. Let $\left(i^{*}, i^{*}+1\right)$ be the peak section in $T_{k_{1}}^{0}$, and the corresponding peak passenger flow be $g\left(i^{*}, k_{1}\right)=\max \left\{g\left(i, k_{1}\right) \mid k_{1}=1,2, \cdots, H_{t}^{0}\right\}$. If the length of period $T_{k_{1}}^{0}$ is $\left|T_{k_{1}}^{0}\right|$, then for $T_{k_{1}}^{0} \in T_{k_{2}}$, the train load factor in flow peak section satisfies

$$
\frac{\varphi_{0} V b d_{k_{2}}\left|T_{k_{1}}^{0}\right|}{\left|T_{k_{2}}\right|} \leq g\left(i^{*}, k_{1}\right) \leq \frac{\varphi_{1} V b d_{k_{2}}\left|T_{k_{1}}^{0}\right|}{\left|T_{k_{2}}\right|} .
$$

The length restriction of the train formation is

$$
\bar{b} \leq b \leq \overline{\bar{b}} \text {, and } b \text { is an integer. }
$$

The train operation periods should satisfy

$$
\begin{aligned}
t_{k}^{b} & =t_{k+1}^{a}, & & k=1,2, \cdots, H_{t}-1 ; \\
t_{k}^{a} & =T_{s}, & & k=1 ; \\
t_{k}^{b} & =T_{e}, & & k=H_{t} .
\end{aligned}
$$

The train frequency should be restricted by train headway $\tau$ [13]. The upper limit of train frequency is

$$
\frac{\left|T_{k}\right|}{\sum_{\substack{l \in U \\ e(i, i+1) \in u_{l}}} d_{k}^{l}} \geq \tau, \quad i=1, \cdots, H_{S-1}, k=1,2, \cdots, H_{t} .
$$

The lowest train frequency for each routing varies. For basic routings, such as long routings, its train frequency should be no less than the minimal operational frequency. In another word, its train operation interval should be no less than the maximum operation interval time $\tau_{0}$. As for non-basic routings, such as short routings, the train frequency should be zero or the train operation interval should not be less than $\tau_{0}$, or equal 0 . The lowest train frequency for each routing is denoted as follows:

$$
\begin{gathered}
\frac{\left|T_{k}\right|}{\left|d_{k}^{l}\right|} \geq \tau_{0}, \quad l \in U_{1}, \\
\frac{\left|T_{k}\right|}{\left|d_{k}^{l}\right|} \geq \tau_{0} \quad \text { or }\left|d_{k}^{l}\right|=0, \quad l \in U_{2},
\end{gathered}
$$

where $U_{1}$ and $U_{2}$ are the sets of basic and non-basic routings.

Each individual passenger will select affordable trains. Thus, the passenger flow distribution will reach user equilibrium state. It must be noted that, there is always stochastic in passenger choice behavior. All in all, the passenger flow of urban rail transit is stochastic equilibrium.

As passenger volume fluctuates with time periods, the passenger choice behavior should be analyzed for each period. We use logit distribution to describe the passenger choice behavior, the probability that passenger flow $f\left(i, j, k_{1}\right)$ selects routing $u_{l_{0}}$ is:

$$
\varphi\left(i, j, k_{1}, l_{0}\right)=\frac{\exp \lambda C\left(i, j, k_{1}, l_{0}\right)}{\sum_{l \in U} \exp \lambda C\left(i, j, k_{1}, l\right)} .
$$

where $\lambda$ is the utility parameter.

The general travel expense of all passengers should be minimized: 


$$
\min Z_{1}=\sum_{l \in U} \sum_{k=1}^{H_{i}^{0}} \sum_{i \in S} \sum_{j \in S} f(i, j, k, l) \cdot C(i, j, k, l)
$$

where the ticket fare and travel time can be viewed as constants, and the passenger travel expense related with the train plan is expressed as

$$
C^{*}(i, j, k, l)=\beta[z(i, j, k)]+\sum_{i^{\prime}=i}^{j-1} y\left(g\left(i^{\prime}, k, l\right)\right) .
$$

Thus, the objective function $Z_{1}$ is simplified as

$$
\min Z_{1}^{\prime}=\sum_{l \in U} \sum_{k=1}^{H_{t}^{0}} \sum_{i \in S} \sum_{j \in S} f(i, j, k, l) \cdot C^{*}(i, j, k, l)
$$

For the service providers, their primary benefits come from ticket income. The train type in the urban rail transit is homogeneous and the ticket fare has no distinction. If the target passenger flow is relatively stable, the ticket income is constant. Let per-train-kilometer cost be $c_{\mathrm{T}}$, and per-car-kilometer cost be $c_{\mathrm{u}}$, then the objective function of the operation cost is

$$
\min Z_{2}=\sum_{k=1}^{H_{t}} \sum_{l=1}^{H_{u}}\left(c_{\mathrm{T}}+b c_{\mathrm{u}}\right) \cdot d_{k}^{l} \cdot w\left(s_{l}, e_{l}\right) .
$$

Maximizing the average load factor is also an important target of the train plan optimization:

$$
\max Z_{3}=\sum_{l \in U} \frac{1}{b V w\left(s_{l}, e_{l}\right)} \sum_{k_{2}=1}^{H_{t_{1}}} \sum_{k_{1}: T_{k_{1}}^{0} \in T_{k_{2}}} \sum_{i=1}^{H_{S}-1} \sum_{j=i+1}^{H_{S}} \frac{\left|T_{k_{2}}\right| w(i, j) \sum_{j=i+1}^{j_{e}} \sum_{i=1}^{i} f\left(i^{\prime}, j^{\prime}, k_{1}, l\right)}{\left|T_{k_{1}}^{0}\right| d_{k_{2}}^{l}} .
$$

The train frequency may vary with the passenger flow. However, if the train frequency is frequently changed, the organization of train operation will be disturbed. Thus the train frequency should keep steady in an operation period as long as possible. And the number of the train operation periods should be minimized, i.e.

$$
\min Z_{4}=H_{t} .
$$

The objective functions (7)-(10) and the constraints (1)-(6) constitute the multi-objective optimization model for the train plan of urban rail transit.

\section{Solution}

The established optimization model can not be solved directly because it is discontinuous, non-differentiable, non-convex and mixed of multi-objective. For this reason, we work out a three-phase solving strategy:

In the first phase, the train formation is optimized.

In the second phase, the train operation period is substituted with passenger travel period, and the train count for each routing is determined.

In the third phase, the strategy of merging train operation periods is designed, and the train frequency is adjusted accordingly.

\subsection{Determining train formation}

The objective functions (7) and (8) are added with the introduction of $\alpha$, the weight factor of the passenger travel expense. The evaluation function of train formation length is

$$
\begin{gathered}
\min Z_{b}=\alpha \sum_{l \in U} \sum_{k=1}^{H_{t}^{0}} \sum_{i \in S} \sum_{j \in S} f(i, j, k, l) \cdot C(i, j, k, l)+ \\
(1-\alpha) \sum_{k=1}^{H_{t}} \sum_{l=1}^{H_{u}}\left(c_{\mathrm{T}}+b c_{\mathrm{u}}\right) \cdot d_{k}^{l} \cdot w\left(s_{l}, e_{l}\right) .
\end{gathered}
$$

The formation length that corresponds to the minimal evaluation function $Z_{b}$ is optimal.

When the train formation length is $b$, the train frequency can be determined by section flow

$$
\sum_{k_{1}: T_{h_{1}}^{0} \in T_{k_{2}}} g\left(i^{*}, k_{2}, l\right)
$$

in the train operation period $k_{2}\left(k_{2}=1,2, \cdots, H_{t}\right)$. Constraint (1) determines a range of $d_{k_{2}}^{l}$ for formation length $b$. Considering the objective function (9), we obtain $d_{k_{2}}^{l}$ :

$$
\begin{aligned}
& \min \left\{d_{k_{2}}^{l} \mid \max \left\{\left(d_{k_{2}}^{l}-1\right) \varphi_{1} V b, d_{k_{2}}^{l} \varphi_{0} V b\right\} \leq\right. \\
& \sum_{k_{1}: T_{1}^{0} \in T_{k_{2}}} g\left(i^{*}, k_{2}, l\right) \leq \\
& \left.\quad \max \left\{d_{k_{2}}^{l} \varphi_{1} V b,\left(d_{k_{2}}^{l}+1\right) \varphi_{0} V b\right\}\right\} .
\end{aligned}
$$

To simplify calculation, each passenger travel period works as one train operation period, and all the trains run from the origin stations to the terminal stations on the urban rail lines. In this way, the solution of formation length can be simplified to meet passenger demands. Every operation period has the train count $d_{k}, k=1,2, \cdots, H_{t}$. The algorithm is described in details below. 


\section{Algorithm 1}

Step 1 Initialization. Let the initial train formation length $b=\bar{b}$, the best train formation solution $b^{*}=b$ and its evaluation function $Z^{*}=+\infty$. Each passenger travel period work as train operation period, $T_{k}=T_{k}^{0}$, $k=1,2, \cdots, H_{t}^{0}$.

Step 2 If $b>\overline{\bar{b}}$, stop.

Step 3 Determine the train count $d_{k}$ in train operation period $T_{k}$. Then examine constraint (4), if $d_{k}>60 / \tau$, $d_{k}=60 / \tau$; examine constraint (5), if $d_{k}<60 / \tau_{0}$, $d_{k}=60 / \tau_{0}$.

Step 4 Calculate the objective function value $Z$ by (11). If $Z<Z^{*}$, then $Z^{*}=Z$, and $b^{*}=b . b=b+1$, go to Step 2.

\subsection{Determining the train frequency}

In this stage, the passenger travel period is still used as train operation period; thus every train operation period has the same passenger flow intensity. To reduce train operation cost, trains operate on the short routing to meet the peak passenger flow.

$$
F_{l_{2}}=\sum_{i, j: e(i, j) \in e\left(s_{l_{2}}, e_{l_{2}}\right)} f\left(i^{*}, l_{2}, k\right)
$$

is the maximum passenger flow on the short routing. The largest train count on the short routing $d U_{k_{2}}^{l_{2}}$ is

$$
\begin{gathered}
\min \left\{d U_{k_{2}}^{l_{2}} \mid \max \left\{\left(d U_{k_{2}}^{l_{2}}-1\right) \varphi_{1} V b, d_{k_{2}}^{l} \varphi_{0} V b\right\} \leq\right. \\
\left.F_{l_{2}} \leq \max \left\{d U_{k_{2}}^{l_{2}} \varphi_{1} V b,\left(d U_{k_{2}}^{l_{2}}+1\right) \varphi_{0} V b\right\}\right\} .
\end{gathered}
$$

Thus, the train count on routing $l_{1}$ is $d_{k_{2}}-d U_{k_{2}}^{l_{2}}$, and on routing $l_{2}$ is $d U_{k_{2}}^{l_{2}}$.

Passengers who choose the short routing can choose the long routing at the same time. An extreme case is that the long routing is the sole selection, i.e. the train counts on long and short routings are $d_{k_{2}}$ and 0 .

The real train count falls between the two extremes, which are determined by the selection behavior of passenger flow:

\section{Algorithm 2}

Step $1 T_{k}=T_{k}^{0}, k=1,2, \cdots, H_{t}^{0}$.

Step 2 Calculate $d U_{k}^{l_{2}}$ by Eq. (13), $d^{\prime}=d U_{k}^{l_{2}}, d^{\prime \prime}=0$.

Step 3 If $d^{\prime}=d^{\prime \prime}$, train count on the long routing is $d_{k}-d^{\prime}$, and that on the short routing is $d^{\prime}$, stop.
Step 4 Let $d_{k}^{l_{1}}=\left(d^{\prime}+d^{\prime \prime}\right) / 2, d_{k}^{l_{2}}=d_{k}-\left(d^{\prime}+d^{\prime \prime}\right) / 2$, and assign passenger flow on the routings according to logit distribution to obtain $g(i, k, l)$.

Step 5 According to the passenger flow distribution, determine the train count on the short routing by Eq. (12). If $d_{k}^{l_{2}} \geq\left(d^{\prime}+d^{\prime \prime}\right) / 2$, let $d^{\prime \prime}=d_{k}^{l_{2}}$; otherwise let $d^{\prime}=d_{k}^{l_{2}}$, and go to Step 3 .

\subsection{Merging train operation periods}

In order for minimization, the operation periods of similar train counts are merged. The train frequency can evaluate the difference of train counts,

$$
\lambda_{k}=\frac{d^{k}}{\left|T_{k}\right|} \quad k=1,2, \cdots, H_{t}
$$

The train count difference in adjacent periods is distinguished by the following criterion:

$$
\delta_{k, k-1}=\sqrt{\sum_{l \in U}\left[\left(\lambda_{k}^{l}-\lambda_{k-1}^{l}\right) / \lambda_{k-1}^{l}\right]^{2}}, \quad k=2,3, \cdots, H_{t} .
$$

The operation periods which have minimal $\delta_{k, k-1}$ will be merged in the first place. The algorithm is expressed as follows:

\section{Algorithm 3}

Step 1 Calculate $\lambda_{k}$ for each train operation period, where $k=1,2, \cdots, H_{t}$.

Step 2 Calculate criterion function $\Delta=\left\{\delta_{k, k-1} \mid k=\right.$ $\left.2,3, \cdots, H_{t}\right\}$ of adjacent periods.

Step 3 If $\Delta=\phi$, stop; otherwise, sort the elements in set $\Delta$ and select the minimal $\delta_{\bar{k}, \bar{k}-1}^{*}$.

Step 4 Assume $\bar{k}$ and $\bar{k}-1$ are objective periods for merging, determine the train counts on different routings by Eq. (12) and make passenger assignment according to logit distribution. Then the feasibility of merging periods is judged by constraint (1). If it is feasible, merge $\bar{k}$ and $\bar{k}-1$ and calculate the new train count $d_{\bar{k}-1}^{l}$, then go to Step 1; otherwise, $\Delta=\Delta \backslash \delta_{\bar{k}, \bar{k}-1}^{*}$, i.e., to eliminate $\delta_{\bar{k}, \bar{k}-1}^{*}$ from $\Delta$, then go to Step 3 .

\section{Case analysis}

We take Changsha Metro Line 2 in the initial operation stage as an example [3]. This is a 28-station line, starting at Zhenqiao Road Station and ending at 
Guangda Station. In addition to the long routing (34.02 $\mathrm{km}$ ) between the start and the end, trains can also operate on the short routing $(21.36 \mathrm{~km})$ between Donglei Road Station and New Changsha Station. $V=220$ person/car, $\bar{b}=4$ and $\overline{\bar{b}}=9$ cars, $\tau=2 \min$ and $\tau_{0}=15$ $\min , \varphi_{0}=0.95, \varphi_{1}=1.4, \alpha=0.5$. And the cost parame- ters of train operation are set as follows: $c_{\mathrm{T}}=60 \mathrm{CNY} /($ train $\cdot \mathrm{km}), \quad c_{\mathrm{u}}=10 \mathrm{CNY} /(\mathrm{car} \cdot \mathrm{km})$. Set $\beta=30 \mathrm{CNY} / \mathrm{h}, T_{s}=6, T_{e}=24$ and $H_{t}^{0}=18 \mathrm{~h}$, meaning the passenger travel period is one hour.

For $T_{k_{1}}^{0} \in T_{k_{2}}$, the congestion cost function of the section $(i, i+1)$ on routing $l$ is

$$
y\left(g\left(i, k_{1}, l\right)\right)=\left\{\begin{array}{cl}
0, & \left|T_{k_{2}}\right| g\left(i, k_{1}, l\right) \leq b V\left|T_{k_{1}}^{0}\right| d_{k_{2}}^{l}, \\
0.15\left(\frac{\left|T_{k_{2}}\right| g\left(i, k_{1}, l\right)}{b V\left|T_{k_{1}}^{0}\right| d_{k_{2}}^{l}}\right)^{4}, & \left|T_{k_{2}}\right| g\left(i, k_{1}, l\right)>b V\left|T_{k_{1}}^{0}\right| d_{k_{2}}^{l} .
\end{array}\right.
$$

With the computer of $1 \mathrm{~GB}$ RAM and $1.7 \mathrm{GHz}$ CPU, the optimization process spent about 2 sec. Fig. 2 shows the relationship between the evaluation function (11) and train formation length. The dotted line shows that the formation length of 4 cars does not meeting transportation capacity. The best formation length is 6 , and the corresponding value of evaluation function is $6809232.5 \mathrm{CNY}$.

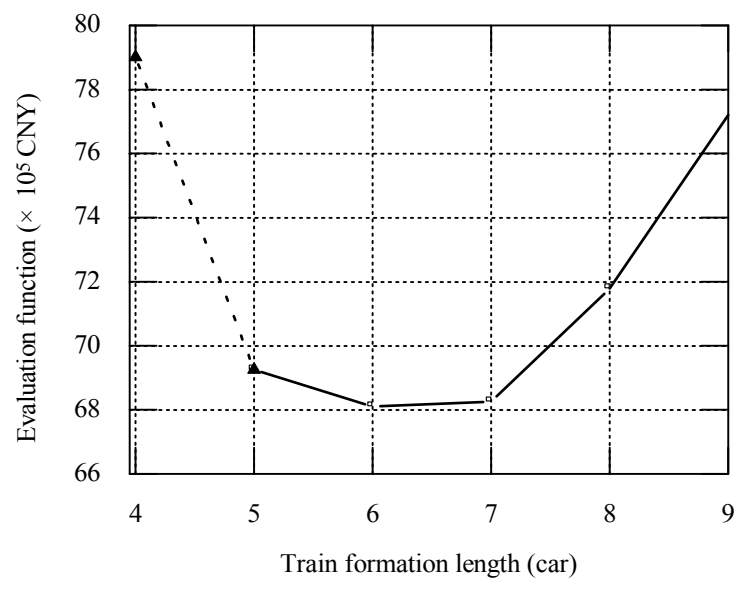

Fig. 2 Solving train formation length

As shown in the Fig. 3, among the total 158 trains, 18 trains run on the long routings, 11 trains on the short routings at the morning peak (from 7 to 8 a.m.), 14 trains run on the long routings, and 4 trains on the short routings at the evening peak (from 17 to 18 p.m.). The operation benefit is $2340275 \mathrm{CNY}$, and the average general travel expense for one passenger is $14.27 \mathrm{CNY}$.

As indicated in Fig. 4, when train operation periods are merged, the train count decreases to 155 . Operation benefit rises to $2364770 \mathrm{CNY}$ with a little increase in average passenger general travel expense, i.e., 14.41 CNY. The average train load factor is $60 \%$. A relative low load factor is because of the unbalanced passenger spatial distribution.

In the model, the weight factor of the passenger travel expense $\alpha$ plays a role in balancing the train operation cost and the passenger travel expense. The train operation cost and average passenger travel expense under different values of $\alpha$ are calculated (see Fig. 5). With the decease of $\alpha$, the passenger travel expense declines, and the train operation cost rises. In general, the value of $\alpha$ is in the range of $0.4-0.6$.

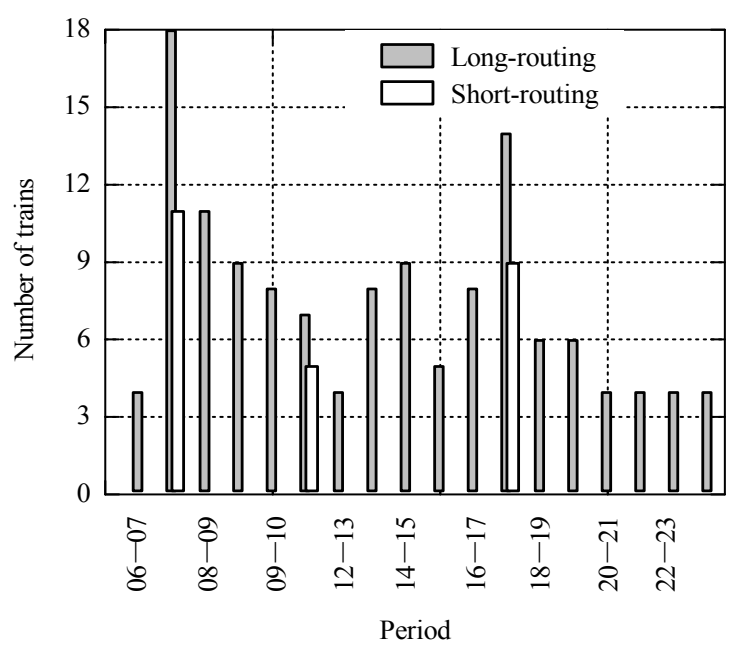

Fig. 3 Train counts in different periods

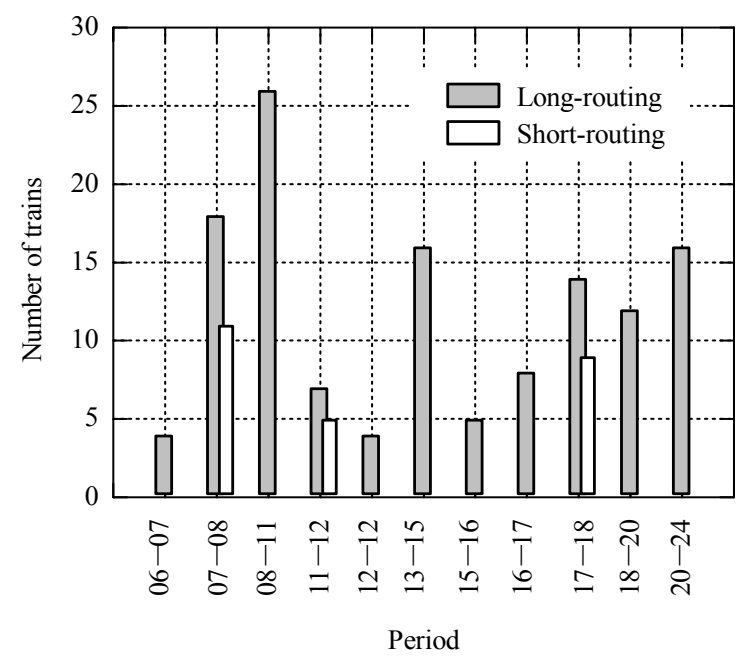

Fig. 4 Final train counts after merging 


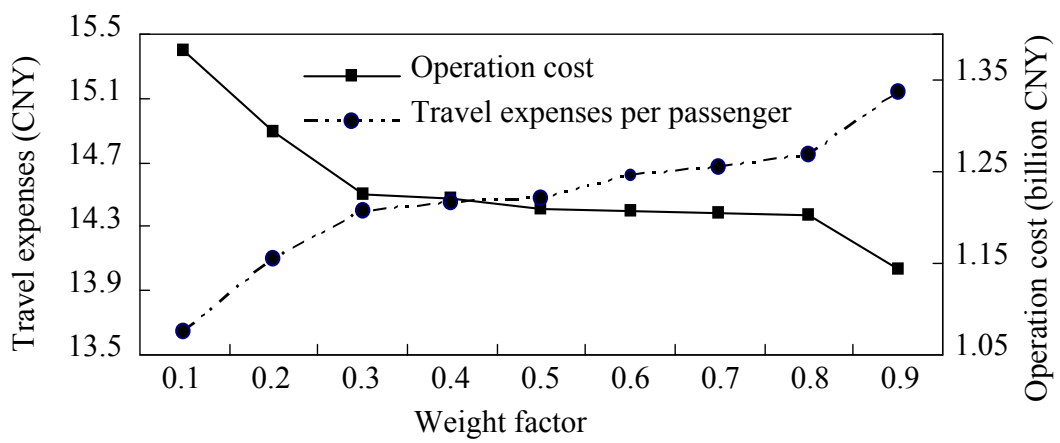

Fig. 5 Effect of weight factor on train plan

\section{Conclusions}

The train plan of urban rail transit under multiple train-routings consists of three components: train formation, train operation periods and correspondingly train counts for each period and train routing. Based on the nested routing mode, we propose a three-phase solving strategy to optimize the train formation, the train count and the operation periods. The method has been used to optimize the train plan of Changsha Metro Line 2, showing satisfactory results. For more complicated routing networks, we may resort to heuristic intelligent algorithm, but the multi-phase solution can still be used.

\section{Acknowledgements}

The work was supported by the National Natural Science Foundation of China (No. 70901076), Research Fund for the Doctoral Program of Higher Education of China (No. 20090162120021) and Natural Science Foundation of Hunan Province (No. 10JJ4046).

\section{References}

[1] B.H. Mao, F. Jiang, Q. Liu, et al., Urban Rail Transit, Beijing: Science Press, 2008 (in Chinese).

[2] R.H. Xu, J.J. Chen, S.M. Du, Study on carrying capacity and use of rolling stock with multi-routing in urban rail transit, Journal of the China Railway Society, 2005, 27(4): 6-10 (in Chinese).

[3] China Railway Siyuan Survey and Design Group Co. Ltd., Feasibility study report of first phase project of
Changsha Metro Line 2, Wuhan: China Railway Siyuan Survey and Design Group Co. Ltd., 2009 (in Chinese).

[4] L.B. Deng, Q. Zeng, W. Gao, et al., Optimization method for train Plan of urban rail transit, Science Paper Online, 2010, 5(10): 767-772 (in Chinese).

[5] A. de Palma, R. Lindsey, Optimal timetable for public transportation, Transportation Research Part B, 2001, 35(8): 89-813.

[6] L.B. Deng, F. Shi, W.L. Zhou, Passenger train plan problem and its advanced research, In: Proc. of the 8th National Youth Conference on Transportation and Communications, Shanghai, 2009: 196-199 (in Chinese).

[7] Y.H. Chang, C.H. Yeh, C.C. Shen, A multi-objective model for passenger train services planning: application to Taiwan's High-Speed Rail Line, Transportation Research Part B, 2000, 34(2): 91-106.

[8] M.R. Bussieck, P. Kreuzer, U.T. Zimmermann, Optimal lines for railway systems, European Journal of Operational Research, 1997, 96(1): 54-63

[9] M.T. Claessens, N.M. van Dijk, P.J. Zwancveld, Cost optimal allocation of rail passenger lines, European Journal of Operational Research, 1998, 110(3): 474-489.

[10] Y. Sun, Q.Z. Shi, Y. Zhao, Method on making train running-plan for urban railway traffic, Journal of Tongji University, 2004, 32(8): 47-49 (in Chinese).

[11] N. Sang, P. Stefano, M. Federico, A modeling framework for passenger assignment on a transport network with timetables, Transportation Science, 2001, 35(3): 238-249.

[12] Q. Tian, H.J. Huang, H. Yang, Equilibrium properties of the morning peak-period commuting in a many-to-one mass transit system, Transportation Research Part B, 2007, 41(6): 616-631.

[13] X.L. Zheng, L. Ma, T. Tang, et al., Analysis of train headway in urban rail transport system, Computer and Communications, 2005, 23(3): 123-125 (in Chinese). 